\title{
BMJ Open Incremental yield of ECG screening repeated annually over 4 years in an adult Japanese population without prior atrial fibrillation: a retrospective cohort study
}

\author{
Yoshiki Nagata (D) , ${ }^{1}$ Takashi Yamagami, ${ }^{1}$ Don Nutbeam, ${ }^{2}$ Ben Freedman, ${ }^{3,4}$ \\ Nicole Lowres ${ }^{3,4}$
}

To cite: Nagata Y, Yamagami T, Nutbeam D, et al. Incremental yield of ECG screening repeated annually over 4 years in an adult Japanese population without prior atrial fibrillation: a retrospective cohort study. BMJ Open 2020;10:e035650. doi:10.1136/ bmjopen-2019-035650

- Prepublication history and additional material for this paper are available online. To view these files, please visit the journal online (http://dx.doi. org/10.1136/bmjopen-2019035650).

Received 10 November 2019 Revised 19 March 2020 Accepted 12 June 2020

\section{Check for updates}

(c) Author(s) (or their employer(s)) 2020. Re-use permitted under CC BY-NC. No commercial re-use. See rights and permissions. Published by BMJ.

${ }^{1}$ Laboratory of Preventive Medicine, Hokuriku Health Service Association, Toyama, Japan

${ }^{2}$ School of Public Health, University of Sydney, Sydney, New South Wales, Australia ${ }^{3}$ Heart Research Institute, Sydney, New South Wales, Australia

${ }^{4}$ Faculty of Medicine, University of Sydney, Sydney, New South Wales, Australia

Correspondence to

Dr Yoshiki Nagata;

drnagata@nsknet.or.jp

\section{ABSTRACT}

Objectives International guidelines recommend opportunistic screening for atrial fibrillation (AF); however, there is no current data to inform how often to repeat screening. We aimed to investigate the incremental annual yield and stroke risk of new AF cases in individuals screened annually over 4 years.

Design A retrospective cohort study.

Setting Hokuriku Health Service Association, Toyama prefecture, Japan.

Participants Employees and their families receiving annual health examinations from Hokuriku Health Service Association.

Intervention Each subject received an annual health examination (including 12-lead ECG) from 2014 to 2017. Only subjects with baseline ECGs in 2012 and/or 2013 were included.

Main outcome measures Rates (cases/100 personyears) of new AF identified each year for 4 consecutive years of screening (stratified according to gender and age groups). Calculated stroke risk of new AF cases using modified $\mathrm{CHA}_{2} \mathrm{DS}_{2}$-VASc scores (without heart failure data) $\left(\mathrm{CHA}_{2} \mathrm{DS}_{2} \mathrm{VASC}=\mathrm{C}\right.$ : congestive heart failure [1 point]; $\mathrm{H}$ : hypertension [1 point]; A2: age 65-74 years [1 point] or age $\geq 75$ years [2 points]; D: diabetes mellitus [1 point]; S: prior stroke or transientischemic attack [2points]; VA: vascular disease [1 point]; and Sc: sex category [female] [1 point])

Results In 2014, 88218 subjects had an ECG (46.8 \pm 12.5 years; $64 \%$ men): identifying $346(0.39 \%)$ known AF and $69(0.08 \%)$ new AF. The incidence rate of new AF increased with age from $0.01 \%(<50$ years) to $0.98 \%(\geq 75$ years) and was higher in men $(0.1 \%)$ than women $(0.05 \%)$. Repeated annual screening over 4 years identified a consistent new AF yield $0.06 \%-0.10 \%$ per year $(0.33 \%-0.55 \% \geq 65$ years). Forty-two per cent of all new AF cases, and $76 \%$ of cases aged $\geq 65$ years, had a class -1 oral anticoagulation (OAC) recommendation (modified $\mathrm{CHA}_{2} \mathrm{DS}_{2}$-VASc score $\geq 2$ men, $\geq 3$ women).

Conclusions Repeated annual ECG screening of the same population provides a consistent yield of new AF each year. The majority of new AF ( $\geq 65$ years) are eligible for anticoagulation for stroke prevention. Although
Strengths and limitations of this study

- This is the first study to investigate the effect on the annual yield of new atrial fibrillation by repeating screening each year in the same population.

- This study uses a large database of more than 140000 individuals attending annual health examinations in Japan.

- The analysis includes more than 57500 people who were screened annually with an ECG for four consecutive years.

- Although the age distribution of the database was skewed towards a younger demographic, the analysis included $\sim 6600$ individuals aged 65 years and over.

AF prevalence and incidence are lower in Japan than Western countries, 2318 new cases would be identified in Toyama prefecture each year with annual screening, of whom 927 would have a high stroke risk with a recommendation for OAC therapy.

\section{INTRODUCTION}

Atrial fibrillation (AF) is a global healthcare problem with evidence suggesting an increasing prevalence and incidence worldwide. ${ }^{1}$ As stroke registries indicate that a third of all ischaemic strokes are AF related, early identification is essential. ${ }^{23}$ Screening people $\geq 65$ years in Western countries, with a single screen, is likely to yield $1.44 \%$ with new $\mathrm{AF}^{4}{ }^{4}$ In Japan, screening is likely to identify a lower incidence of AF; however, those identified have a high relative risk of stroke $(1.61 ; 95 \%$ CI 0.50 to 5.16) and all-cause mortality (1.71; $95 \%$ CI 1.20 to 2.43$){ }^{5}$

International guidelines recommend opportunistic screening for AF in the clinic by pulse palpation in people aged $\geq 65$ years to reduce AF-related strokes. ${ }^{67}$ The Wilson 


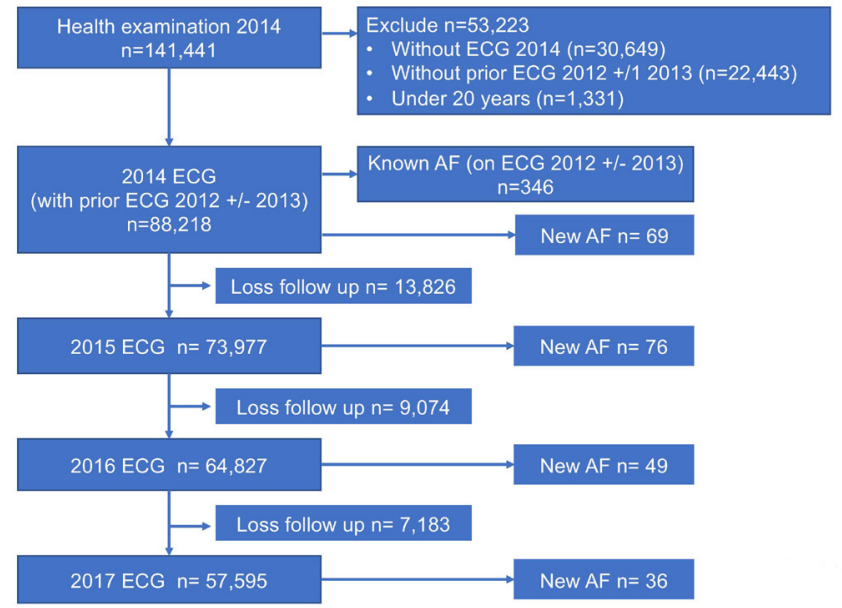

Figure 1 Study flowchart. AF, atrial fibrillation.

and Jungner, WHO, criteria for a screening test requires that any screening intervention must have a defined timeframe for when screening should be repeated and that it is not a once-off process. ${ }^{8}$ However, there is no clear recommendation in the AF guidelines regarding if screening should be once only or repeated, and what the frequency for repetition of screening should be ${ }^{67}$ To date, there are no published data regarding the incremental yield that would be found by repeating opportunistic or systematic screening each year in people without AF, and it is uncertain whether there would be diminishing returns from screening with each subsequent year. In order to determine the incremental yield of repeated screening, physicians need to repeat screening in an AF-negative population on a regular basis.

In Japan, employers have a legal obligation to provide annual health examinations for the employees and their families. For non-employed or retired residents, the government provides access to annual health examinations, although attendance is optional. The annual health examination screen is comprehensive, including screening for respiratory, metabolic, cardiovascular, ophthalmology, hearing, gynaecology and lifestyle disorders, with many companies, including a 12-lead ECG. We had the unique opportunity to investigate the effect of repeating screening each year on the yield of new AF, by assessing a large database of annual ECGs taken during health examinations in Toyama prefecture, Japan. Therefore, this study aimed to: (1) investigate the incremental yield of new AF identified through annual screening repeated over a 4-year period; (2) determine the stroke risk of new AF cases identified through screening and (3) determine the estimated prevalence of AF in Toyama prefecture, Japan.

\section{METHODS}

This retrospective cohort study examined data from annual health examinations performed in Toyama prefecture by the Hokuriku Health Service Association between 2012 and 2017.

\section{Annual health examination}

Hokuriku Health Service Association in Toyama prefecture performs annual health examinations on approximately 150000 workers and their families each year. The annual health examination includes a 12-lead ECG, chest X-ray, blood pressure measurement, body mass index, blood sugar, HbA1c (hemoglobin A1c), cholesterol (total cholesterol, low density lipoprotein, triglyceride, high density lipoprotein), uric acid, liver enzyme (aspartate aminotransferase, alanine aminotransferase, gammaglutamyl transpeptidase), renal function (blood urea nitrogen, creatinine), urinalysis and testing for blood cell count and blood chemistry. The examination also comprises a self-reported health questionnaire which includes information on the previous history of stroke, diabetes mellitus, hypertension, myocardial infarction, angina pectoris and arrhythmia.

Hypertension was diagnosed if peripheral blood pressure was $\geq 140 / 90 \mathrm{~mm} \mathrm{Hg},{ }^{9}$ or if the health questionnaire indicated current treatment for hypertension. Diabetes was diagnosed using $\mathrm{HbA1c} \geq 6.5 \%$ (National Glycohemoglobin Standardization Program), a fasting blood glucose concentration of $\geq 126 \mathrm{mg} / \mathrm{dL}$, or a random blood glucose concentration of $\geq 200 \mathrm{mg} / \mathrm{dL},{ }^{10}$ or if the health questionnaire indicated current treatment for diabetes. AF was diagnosed from the 12-lead ECG by a trained cardiology doctor in Hokuriku Health Service Association: AF (Minnesota Codes 8-3-1 and 8-3-3) or atrial flutter (Minnesota Codes 8-3-2 and 8-3-4). AF symptoms were determined from information contained in the health questionnaire. AF symptoms were classified into typical symptoms (palpitations or erratic pulse), atypical symptoms (dizziness, orthostatic dizziness, dyspnoea and chest pain) or asymptomatic (ie, no symptoms).

\section{Study subjects}

Data were retrieved from electronic records for all 141441 people who attended for a health examination in 2014: a 12-lead ECG was performed in 110792 of the examinations. Only people aged $\geq 20$ years, and with a prior ECG recorded in 2012 or 2013, were deemed eligible; therefore, the baseline sample consisted of 88218 subjects (figure 1). Study subjects with AF diagnosed on ECG in either 2012 or 2013 were considered to have 'known AF', which is likely to be mainly permanent and persistent forms of AF. All subjects without known AF were followed up over 4 years, from 2014 until 2017, with consecutive health examinations, including an annual ECG. The annual incidence (cases per 100 person-years) of newonset AF was calculated for each year (2014-2017) and stratified according to gender and age groups.

\section{Modified $\mathrm{CHA}_{2} \mathrm{DS}_{2}$-VASc score}

An estimation of stroke risk (ie, modified $\mathrm{CHA}_{2} \mathrm{DS}_{2}$-VASc score) was calculated without points for heart failure or peripheral artery disease, as health examination data were not available for the presence of either of these co-morbidities $\left(\mathrm{CHA}_{2} \mathrm{DS}_{2} \mathrm{VASc}=\mathrm{C}\right.$ : congestive heart 


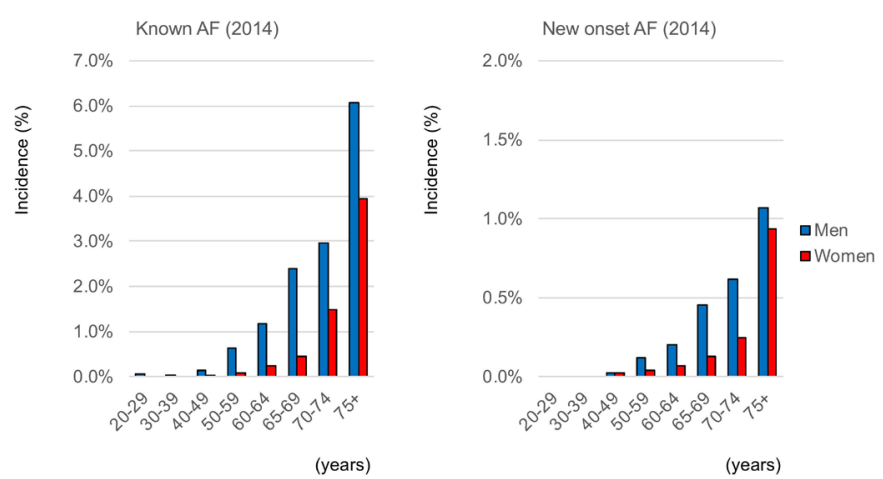

Figure 2 Yield of known and new-onset AF stratified by age and gender. AF, atrial fibrillation.

failure [1 point]; $\mathrm{H}$ : hypertension [1 point]; A2: age 6574 years [ 1 point] or age $\geq 75$ years [2 points]; D: diabetes mellitus [1 point]; $\mathrm{S}$ : prior stroke or transientischemic attack [2points]; VA: vascular disease [1 point]; and Sc: sex category [female] [1 point]). The modified $\mathrm{CHA}_{2} \mathrm{DS}_{2}-$ VASc score was calculated for known AF using 2014 data and for new AF using data from the year of detection. Subjects were categorised according to guideline recommendations for oral anticoagulation (OAC): "no OAC recommendation' (modified $\mathrm{CHA}_{2} \mathrm{DS}_{2}$-VASc score: 0 men, 1 women); 'consider OAC' (modified $\mathrm{CHA}_{2} \mathrm{DS}_{2}$ VASc score: 1 men, 2 women) or 'class-1 OAC recommendation' (modified $\mathrm{CHA}_{2} \mathrm{DS}_{2}$-VASc score: $\geq 2$ men, $\geq 3$ women) ${ }^{6}$

\section{Prevalence of AF in Toyama prefecture}

The estimated prevalence of AF, in the Toyama prefecture, was calculated using the rates of known and new AF detected in our study in 2014 and assuming that the rate is constant. These rates were extrapolated to the 2014 Toyama population data sourced from the Toyama prefectural government. ${ }^{11}$ The data were stratified according to age and gender-specific prevalence, using age-stratified and gender-stratified results from our study (online supplementary 1) and the 2014 Toyama population data. ${ }^{11}$

\section{Statistical analysis}

Continuous variables are presented as mean $\pm \mathrm{SD}$ and categorical variables as numbers and percentages. The yield of known $\mathrm{AF}$ and new $\mathrm{AF}$ detected through screening are presented as rates (ie, cases per 100 person-years) with accompanying binomial 95\% CI calculated using ClopperPearson methodology. AF detection rates were stratified according to age groups and gender. Estimated AF prevalence rates are also presented as rates (cases per 100 person-years). Logistic regression, linear regression and Pearsons $\chi^{2}$ test were used to assess time trends for the yield of new AF detected each year. Data were collected and analysed by the Statistical Package for Social Sciences software (IBM SPSS Statistics V.25).
Table 1 Characteristics of eligible cohort in 2014

\begin{tabular}{lc} 
Characteristics & Cohort $(\mathbf{n = 8 8 2 1 8})$ \\
\hline Age, years, mean \pm SD & $46.8 \pm 12.5$ \\
Age 65-74 years, $\mathrm{n}(\%)$ & $5874(6.7)$ \\
Age 75 and over years, $\mathrm{n}(\%)$ & $814(0.9)$ \\
Gender, men, $\mathrm{n}(\%)$ & $56781(64)$ \\
Co-morbidities & \\
Hypertension & $10771(12)$ \\
Diabetes mellitus & $3597(4.1)$ \\
Previous cerebrovascular disease & $1029(1.2)$ \\
Myocardial infarction & $335(0.4)$ \\
Angina pectoris & $541(0.6)$ \\
\hline
\end{tabular}

\section{Patient and public involvement}

There were no funds or time allocated for patient and public inolvement so we were unable to involve patients. We have invited patients to help us develop our dissemination strategy.

\section{RESULTS}

The eligible cohort included 88218 people, ranging in age from 20 to 109 years (mean age $46.8 \pm 12.5$ years), of which $92 \%$ were $<65$ years old and $64 \%$ were male (table 1, online supplementary 2). Table 1 outlines the characteristics of the eligible cohort. In 2014, the rate of known $\mathrm{AF}$ was $0.39 \%$ (95\% CI $0.35 \%$ to $0.44 \%$ ) ( $\mathrm{n}=346)$, mean age $62.9 \pm 11.4$ years (range 22-103 years) (figure 2, online supplementary 1). The sex-specific rate of known AF was $0.52 \%$ (95\% CI $0.46 \%$ to $0.58 \%$ ) in men and $0.16 \%(95 \%$ CI $0.12 \%$ to $0.21 \%)$ in women. From the 2014 ECG, new-onset AF was identified in $0.08 \%$ (95\% CI $0.06 \%$ to $0.10 \%)(\mathrm{n}=69)$, mean age $64.0 \pm 12.6$ years (range 43-109 years) (figure 2, online supplementary $1)$. The sex-specific incidence rate of new-onset $\mathrm{AF}$ was $0.10 \%$ (95\% CI $0.07 \%$ to $0.12 \%$ ) in men and $0.05 \%$ (95\% CI $0.03 \%$ to $0.08 \%$ ) in women. The rate of both known and new onset AF increased with age and was higher in men than women. From the 6688 subjects aged 65 years and over in 2014, there were 149 known AF cases (2.23\%, $95 \%$ CI $1.89 \%$ to $2.61 \%$ ) and 30 new-onset $\mathrm{AF}$ cases $(0.45 \%, 95 \%$ CI $0.30 \%$ to $0.64 \%)$.

For the 2014 cohort with known AF, 51\% (175/346 cases) had hypertension for which $77 \%$ were on medication and 22\% (77/346 cases) had diabetes for which $56 \%$ were on medication. In those with new-onset AF in 2014, hypertension was present in $45 \%$ (31/69 cases) with $77 \%$ on medication and diabetes was present in $16 \%(11 / 69$ cases) for which $45 \%$ were taking medication.

\section{Incremental yield of annual screening}

The incremental yield of new-onset AF identified through repeat annual ECG screening of the same cohort with no prior history of AF is outlined in figure 1. Of the 88218 people screened in 2014, 73977 were screened again in 


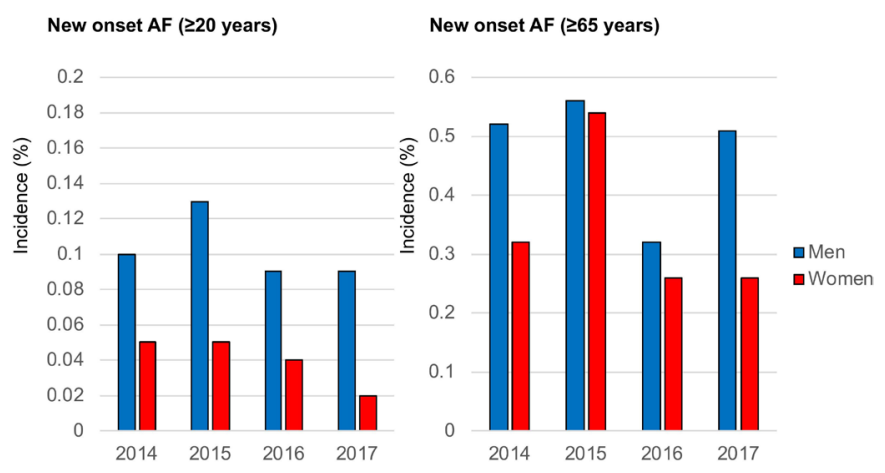

Figure 3 Incidence of new-onset AF detected each year from 2014 to 2017. AF, atrial fibrillation.

2015; 64827 had repeat screens in both 2015 and 2016 and 57595 had repeat screens in 2015, 2016 and 2017. Therefore, the follow-up rate for all 4 years was $65 \%$ of the baseline population.

Yield of new AF did not appear to decline with each consecutive year of screening from 2014 to 2017 (0.08\% (95\% CI $0.06 \%$ to $0.10 \%), 0.10 \%$ (95\% CI $0.08 \%$ to $0.13 \%$ ), $0.08 \%$ (95\% CI $0.06 \%$ to $0.10 \%$ ) and $0.06 \%(95 \%$ CI $0.04 \%$ to $0.09 \%$ ) in each year, respectively) (online supplementary 3 ). The incremental yield was notably and consistently higher in men than women (figure 3). The mean incidence rates of new-onset $\mathrm{AF}$ over the 4 years were $0.10 \%$ in men and $0.04 \%$ in women.

When focusing the results to subjects aged 65 years and over, in line with guideline recommendations for $\mathrm{AF}$ screening, the screening yield was higher than that for the general population. The incidence rate remained relatively constant over the four consecutive years of screening and was $0.45 \%$ (95\% CI $0.30 \%$ to $0.64 \%), 0.55 \%$ (95\% CI $0.36 \%$ to $0.80 \%), 0.33 \%$ (95\% CI $0.18 \%$ to $0.57 \%$ ) and $0.42 \%$ (95\% CI $0.22 \%$ to $0.71 \%$ ), respectively, with no noticeable decline over time (online supplementary 3). Overall, there was a higher yield for men than women despite a reduction in the incidence rate for men in the
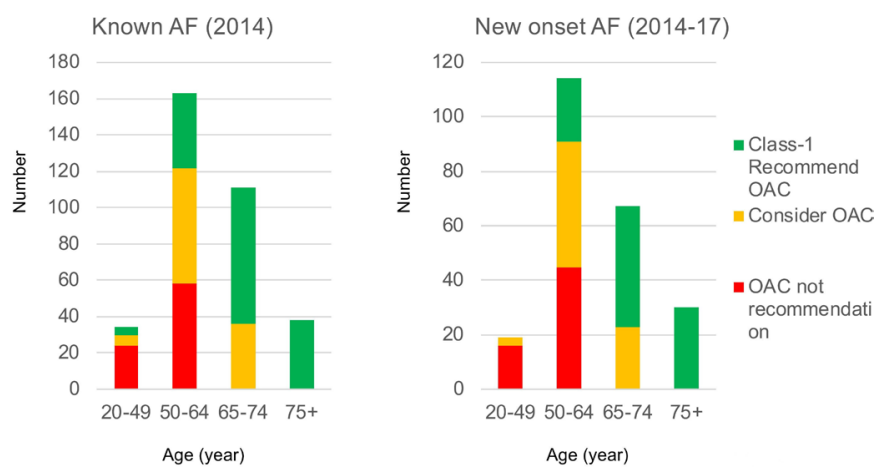

Figure 4 Oral-anticoagulation recommendations. AF, atrial fibrillation; OAC, oral-anticoagulation therapy. Note. Modified $\mathrm{CHA}_{2} \mathrm{DS}_{2}$-VASc scores are likely an underestimation as scores were calculated from health examination data, which did not include information on heart failure or peripheral artery disease. third year of screening (figure 3). The mean incidence rates were $0.48 \%$ in men and $0.37 \%$ in women.

In total, 230 cases of new-onset AF were identified over the 4 years. Most of these cases were asymptomatic $73 \%(\mathrm{n}=167)$. Typical symptoms were reported by only $20 \%(\mathrm{n}=45)$ and atypical symptoms were reported by $8 \%$ $(\mathrm{n}=18)$.

\section{Stroke risk and oral anticoagulation eligibility}

As anticipated, mean modified $\mathrm{CHA}_{2} \mathrm{DS}_{2}$-VASc scores increased with age, reaching $4.1 \pm 1.5$ for known $\mathrm{AF}$ and $3.7 \pm 1.2$ for new AF in those aged $\geq 75$ years (online supplementary 4). Almost half $(\mathrm{n}=255,44 \%)$ of all $\mathrm{AF}$ cases had a class-1 recommendation for OAC: $46 \%(\mathrm{n}=158)$ of known $\mathrm{AF}$ and $42 \%(\mathrm{n}=97)$ of new-onset $\mathrm{AF}$ (figure 4). When considering only subjects $\geq 65$ years $(n=246)$, the number with a class-1 OAC recommendation rose significantly to $76 \%(\mathrm{n}=187)$ of the population: $76 \%(113 / 149)$ of known $\mathrm{AF}$ and $76 \%$ (74/97) of new-onset $\mathrm{AF}$ (figure 4).

\section{Estimated AF prevalence in Toyama prefecture}

The number of people with known AF in Toyama prefecture in 2014 was estimated to be 11212 (6351 men, 4861 women), based on the age-specific and sex-specific rates of known and new AF in the study cohort in 2014, and assuming that the rate is constant (online supplementary $5)$. This equates to an overall AF prevalence of $1.53 \%$ for adults $\geq 20$ years old in the Toyama prefecture: $1.79 \%$ prevalence for men and $1.30 \%$ prevalence for women. As expected, numbers of known AF increase with increasing age in both men and women. Each year, there is likely to be an additional 2318 new cases of AF (1165 men, 1154 women). Due to the large population of women over 75 years in Toyama prefecture, almost half of all new AF cases are likely to be women aged $\geq 75$ years, who all have a class-1 OAC recommendation.

\section{DISCUSSION}

To our knowledge, this is the first study to report the incremental yield of new AF identified from repeating screening each year in a population without prior AF. Our study in Toyama prefecture, Japan, identified that the yield of new AF ranged between $0.06 \%$ and $0.1 \%$ each year (for the cohort $\geq 20$ years), and did not appear to decline over time during the 4-year follow-up period. When limiting the results to subjects aged 65 years and over, as per guideline recommendations for AF screening, the annual yield of new AF ranged between $0.33 \%$ and $0.55 \%$ each year, and again, did not appear to decline over the 4-year period. Across all age groups, the incremental yield of new AF was greater in men than in women, in keeping with the higher prevalence in men than women. It is important to note that $80 \%$ of the new AF cases aged 65 years and over had a sufficient stroke risk to receive a class-1 recommendation for OAC. This is likely an underestimation of the actual number eligible for OAC as we were unable to assign points to the $\mathrm{CHA}_{2} \mathrm{DS}_{2}$-VASc scores 
for heart failure or peripheral artery disease due to the absence of these data. The high proportion of treatable $\mathrm{AF}$ indicates that the annual screen-detected $\mathrm{AF}$ is of great clinical relevance and, with appropriate antithrombotic therapy, would be expected to reduce cardioembolic stroke due to unknown AF.

It is well established that significant racial differences exist, and rates of AF in Japan are much lower across all age groups, compared with representative data reported from Western countries. ${ }^{12}{ }^{13}$ It is therefore not surprising that the incidence rate of new $\mathrm{AF}$ for people aged $\geq 65$ years is also lower in our study $(\sim 0.45 \% /$ year $)$ than the rate of $1.4 \%$ in Western Countries, identified in a systematic review of screening. ${ }^{14}$ It is thought that lower AF rates in Japan may be related to overall lower incidences of cardio-metabolic and other risk factors for AF, but over the next few decades, AF prevalence may increase with a gradual increase in lifestyle-related cardio-metabolic risk factors. ${ }^{12}{ }^{13}$ Therefore, the incidence of new AF in Japan may escalate in the future and will benefit from early detection. However, it is likely that many cases would not be detected without annual screening, as the majority $(\sim 73 \%)$ of our new AF cases were asymptomatic. This high rate of asymptomatic $\mathrm{AF}$ is well documented across many countries, supporting the need to screen. ${ }^{15}$ An American study interrogating pacemaker data has shown that palpitations occur in only $30 \%$ of all AF episodes, and a study using data from the Fushimi AF registry, Japan, has also reported that $50.3 \%$ patients with $\mathrm{AF}$ were asymptomtic. ${ }^{16}$

As annual health checks, including an ECG, are available to all residents, early detection of new-onset $\mathrm{AF}$ should be possible on a population-wide level in Japan. Annual health checks are comparable in some ways to systematic screening due to their organised nature; however, it also differs from systematic $\mathrm{AF}$ screening as it is not targeted at the desired age-group $\geq 65$ years, testing is not focused on detecting $\mathrm{AF}$, and it lacks a clear and established treatment/management pathway should AF be detected. Nonetheless, as detection rates are known to be the same for both opportunistic and systematic screening, ${ }^{17}$ it is likely that our results could be valid for both methods.

The constant yield identified each year through screening is important in terms of guiding screening recommendations and indicates repeating screening on an annual basis may be viable, at least over 4 years. Data from our study can be used to inform and model cost-effectiveness analyses of repeating single-timepoint ECG screening annually. However, the likely costs and potential benefits of repeating screening each year will need to be individually assessed for each setting in relation to the prevalence of $\mathrm{AF}$ in the local population and the method of screening, especially in countries where annual health checks (including 12-lead ECG) are not an established practice. Many studies have demonstrated the cost-effectiveness of AF single-timepoint screening in various settings, including general practice and community in Western countries. ${ }^{17-21}$ In Japan, both annual
ECG screening and annual pulse palpation screening programmes have been deemed to be cost-effective for stroke prevention, when calculated using a Markov modelling programme, regardless of the lower incidence of new AF in Japanese people. ${ }^{22}$

In our study, the rate of known $\mathrm{AF}(0.39 \%)$ was lower than the rates reported in earlier studies in Japan, whose cohorts had very different characteristics. Our study subjects were younger (age $\geq 20$ years; mean 47 years) than other studies; and the majority of subjects were healthy workers and their families. A study of registry data in 2009 identified a higher $\mathrm{AF}$ prevalence rate of $0.56 \%$ using health examinations from a mixture of company clinics, health centres and local governments in Japan, in adults aged $\geq 40$ years, with the majority ( $73 \%$ ) of the cohort aged 50 years and over. ${ }^{23}$ In a second study using annual health examination data of non-employee residents in local government, the AF prevalence rate was higher again at $1.6 \%$ for adults aged $\geq 40$ years (mean age $\sim 72 \pm 10$ years).$^{24}$ As local governments provide annual health examinations for retired workers (retirement occurs at $\sim 60-65$ years of age) and those unable to work, studies using local government health examinations are likely to have a more elderly or unwell population than our study. However, when considering just the population over 65 years, our data suggest an overall AF prevalence (known plus new AF) of $\sim 2.7 \%$ which is in line with rates from other Japanese studies reporting a prevalence of $1.0 \%-1.5 \%$ for ages $60-69$ years; $2.9 \%$ for age $70+$ years and $3.2 \%$ for age $80+$ years. ${ }^{23-25}$

Our data indicate that there will be 2318 new cases of AF in Toyama prefecture each year, and $40 \%$ will have a high risk of stroke and a guideline recommendation for OAC. In current practice, OAC prescription is low: the reported OAC prescription rate from the Fushimi AF Registry was $55 \%$ despite a mean $\mathrm{CHA}_{2} \mathrm{DS}_{2}$-VASc score of $3.4,{ }^{26}$ and the Shinken Database reported OAC prescription rates of $46 \%{ }^{5}$ Furthermore, analysis of the J-Rhythm registry, Fushimi AF registry, and the Shinken database identified a high rate of stroke (18.4 per 1000 person-years) in AF patients with a $\mathrm{CHA}_{2} \mathrm{DS}_{2}-\mathrm{VASc}$ score $\geq 2$ not receiving OAC. ${ }^{27}$ In a retrospective study of Danish patients with $\mathrm{AF}, 39 \%$ received antiplatelet therapy alone, and 25\% patients with $\mathrm{AF}$ did not receive any antithrombotic therapy before the stroke. ${ }^{28}$ It is, therefore, vital that guideline-based OAC prescription is improved. To compound this issue, there is no current defined pathway for medical review if new AF is identified through annual health checks in Japan. Therefore, there is a need to establish viable treatment pathways for those identified with new $\mathrm{AF}$, to ensure appropriate access to medical review and evidence-based OAC treatment to prevent stroke.

\section{Limitations}

Although our analysis was based on 88218 people screened, the distribution of the sample was skewed towards a younger demographic with a mean age of 47 years, and the primary age of interest in AF screening is 
generally $\geq 65$ years. However, it is important to note that our data contains $\sim 6600$ people over 65 years, and there are few other screening studies who have managed to screen more than 4000 people over the age of 65 years. ${ }^{4}$ As a single ECG screen was performed each year, it is likely that some people with paroxysmal AF may not have been identified, and those who were identified most likely had permanent and persistent forms of AF. Consequently, our baseline sample may contain some people with undetected paroxysmal $\mathrm{AF}$, and it is possible that our results may underestimate the prevalence of AF in the Toyama prefecture. Furthermore, as we did not have data on heart failure and peripheral artery disease in the study dataset, we presented a modified $\mathrm{CHA}_{2} \mathrm{DS}_{2}$-VASc score. The modified score will likely underestimate the overall stroke risk to a small degree, meaning our results should be considered a conservative estimate of stroke risk. Our data were collected from one site in the Toyama prefecture, Japan, and therefore may not be generalisable to other prefectures in Japan or other countries.

\section{CONCLUSION}

Repeating screening on an annual basis seems likely to yield a relatively consistent rate of $\mathrm{AF}$ each year, at least over 4 years of follow-up in Japan. Incidence of new AF, and overall AF prevalence, is lower in Japan than Western countries, but the majority of new AF cases identified (aged 65 years and over) have a guideline OAC recommendation for stroke prevention. Annual ECG screening in this population is likely to identify an incremental yield of $\sim 0.08 \%$ with unknown $\mathrm{AF}$, equating to 2318 new cases annually in Toyama prefecture, Japan. It is important to have a viable treatment pathway in place for these newly identified AF cases, to ensure appropriate access to medical review and evidence-based OAC treatment to prevent stroke. The potential application of annual AF screening to other countries where systematic annual health screening, including an ECG, is not established practice would need careful examination in relation to likely cost and potential benefit.

Acknowledgements The authors would like to acknowledge the expert statistical assistance of Hongbing Wang for the analyses in this study.

Contributors YN, TY, DN, SBF and NL designed the study. YN and TY gained ethical approval and data collection. All authors contributed to data analysis. YN drafted the manuscript. All authors reviewed and approved the final manuscript.

Funding NL is funded by a NSW Health Early Career Fellowship (H16/52 168).

Competing interests SBF reports grants, personal fees and non-financial support from Bayer, grants, personal fees and non-financial support from BMS-PFizer, personal fees and non-financial support from Daiichi-Sankyo, outside the submitted work.

Patient and public involvement Patients and/or the public were not involved in the design, or conduct, or reporting, or dissemination plans of this research.

Patient consent for publication Not required.

Ethics approval Ethics approval has been granted through the Hokuriku Health Service Association Ethics Committee.

Provenance and peer review Not commissioned; externally peer reviewed.
Data availability statement Data may be obtained from a third party and are not publicly available.

Open access This is an open access article distributed in accordance with the Creative Commons Attribution Non Commercial (CC BY-NC 4.0) license, which permits others to distribute, remix, adapt, build upon this work non-commercially, and license their derivative works on different terms, provided the original work is properly cited, appropriate credit is given, any changes made indicated, and the use is non-commercial. See: http://creativecommons.org/licenses/by-nc/4.0/.

ORCID iD

Yoshiki Nagata http://orcid.org/0000-0002-0217-697X

\section{REFERENCES}

1 Chugh SS, Havmoeller R, Narayanan K, et al. Worldwide epidemiology of atrial fibrillation: a global burden of disease 2010 study. Circulation 2014;129:837-47.

2 Friberg L, Rosenqvist M, Lindgren A, et al. High prevalence of atrial fibrillation among patients with ischemic stroke. Stroke 2014;45:2599-605

3 Björck S, Palaszewski B, Friberg L, et al. Atrial fibrillation, stroke risk, and warfarin therapy revisited: a population-based study. Stroke 2013;44:3103-8.

4 Lowres N, Olivier J, Chao T-F, et al. Estimated stroke risk, yield, and number needed to screen for atrial fibrillation detected through single time screening: a multicountry patient-level meta-analysis of 141,220 screened individuals. PLoS Med 2019;16:e1002903.

5 Suzuki S, Yamashita T, Otsuka T, et al. Recent mortality of Japanese patients with atrial fibrillation in an urban city of Tokyo. J Cardiol 2011;58:116-23.

6 Kirchhof P, Benussi S, Kotecha D, et al. 2016 ESC guidelines for the management of atrial fibrillation developed in collaboration with EACTS. Eur Heart J 2016;37:2893-962.

7 Hobbs FR, Taylor CJ, Jan Geersing G, et al. European primary care cardiovascular society (EPCCS) consensus guidance on stroke prevention in atrial fibrillation (SPAF) in primary care. Eur J Prev Cardiol 2016;23:460-73.

8 Wilson JMG, Jungner G. Principles and practice of screening for disease. World Health Organization, Public Health papers, 1968. http://www.who.int/iris/handle/10665/37650

9 Shimamoto K, Ando K, Fujita T, et al. The Japanese society of hypertension guidelines for the management of hypertension (JSH 2014). Hypertens Res 2014;37:253.

10 Haneda M, Noda M, Origasa H, et al. Japanese clinical practice guideline for diabetes 2016. Diabetol Int 2018;9:1-45.

11 Toyama government. Toyama prefecture population data. Available: http://www.pref.toyama.jp/sections/1015/lib/jinko/index_h26.html [Accessed 20 Mar 2019].

12 Gavino Al, McLachlan CS. Review of screening studies for atrial fibrillation in rural populations of 11 countries. Proc 2017;30:280-5.

13 Kodani E, Atarashi H. Prevalence of atrial fibrillation in Asia and the world. J Arrhythm 2012;28:330-7.

14 Lowres N, Neubeck L, Redfern J, et al. Screening to identify unknown atrial fibrillation. a systematic review. Thromb Haemost 2013;110:213-22.

15 Freedman B, Camm J, Calkins $\mathrm{H}$, et al. Screening for atrial fibrillation Circulation 2017;135:1851-67.

16 Akao M, Chun Y-H, Wada $\mathrm{H}$, et al. Current status of clinical background of patients with atrial fibrillation in a community-based survey: the Fushimi AF registry. J Cardiol 2013;61:260-6.

17 Fitzmaurice DA, Hobbs FDR, Jowett S, et al. Screening versus routine practice in detection of atrial fibrillation in patients aged 65 or over: cluster randomised controlled trial. BMJ 2007;335:383-6.

18 Lowres N, Neubeck L, Salkeld G, et al. Feasibility and costeffectiveness of stroke prevention through community screening for atrial fibrillation using iPhone ECG in pharmacies. The SEARCH-AF study. Thromb Haemost 2014;111:1167-76.

19 Jacobs MS, Kaasenbrood F, Postma MJ, et al. Cost-effectiveness of screening for atrial fibrillation in primary care with a handheld, single-lead electrocardiogram device in the Netherlands. Europace 2018;20:12-18.

20 Welton NJ, McAleenan A, Thom HH, et al. Screening strategies for atrial fibrillation: a systematic review and cost-effectiveness analysis. Health Technol Assess 2017;21:1-236.

21 Tarride J-E, Quinn FR, Blackhouse G, et al. Is screening for atrial fibrillation in Canadian family practices cost-effective in patients 65 years and older? Can J Cardiol 2018;34:1522-5. 
22 Maeda K, Shimbo T, Fukui T. Cost-effectiveness of a communitybased screening programme for chronic atrial fibrillation in Japan. $J$ Med Screen 2004;11:97-102.

23 Inoue $\mathrm{H}$, Fujiki A, Origasa $\mathrm{H}$, et al. Prevalence of atrial fibrillation in the general population of Japan: an analysis based on periodic health examination. Int J Cardiol 2009;137:102-7.

24 Iguchi Y, Kimura K, Aoki J, et al. Prevalence of atrial fibrillation in community-dwelling Japanese aged 40 years or older in Japan: analysis of 41,436 non-employee residents in Kurashiki-city. Circ $J$ 2008:72:909-13.

25 Ohsawa M, Okayama A, Sakata K, et al. Rapid increase in estimated number of persons with atrial fibrillation in Japan: an analysis from national surveys on cardiovascular diseases in 1980, 1990 and 2000 $J$ Epidemiol 2005;15:194-6.

26 An Y, Ogawa H, Yamashita Y, et al. Causes of death in Japanese patients with atrial fibrillation: the Fushimi atrial fibrillation registry. Eur Heart J Qual Care Clin Outcomes 2019;5:35-42.

27 Suzuki S, Yamashita T, Okumura K, et al. Incidence of ischemic stroke in Japanese patients with atrial fibrillation not receiving anticoagulation therapy--pooled analysis of the Shinken Database, J-RHYTHM Registry, and Fushimi AF Registry. Circ J 2015;79:432-8.

28 Gundlund A, Xian Y, Peterson ED, et al. Prestroke and poststroke antithrombotic therapy in patients with atrial fibrillation: results from a nationwide cohort. JAMA Netw Open 2018;1:1-12. 